\title{
IDENTIFICATION OF SEMICONDUCTOR CONTACT RESISTIVITY
}

\author{
BY \\ STAVROS BUSENBERG (Harvey Mudd College, Claremont, California) \\ AND \\ WEIFU FANG (Claremont Graduate School, Claremont, California)
}

1. Introduction. In a VLSI semiconductor component, a contact is the place where the semiconductor is attached to a metal layer, serving as an access to the semiconductor. The main parameter that describes the quality of the contact interface is its resistivity $\rho_{c}$. It is well known that $\rho_{c}$ becomes a dominant design factor as components reduce to submicron size, so it is important to obtain accurate values of $\rho_{c}$. Due to the complexity of manufacture and miniaturization, it is impossible to directly measure the contact resistivity and to exactly control the location of the contact window. In order to estimate $\rho_{c}$ and detect the location of the contact window, current is applied to the component and voltage is measured at some accessible place far away from the contact window. There are extensive experimental and computer simulations on this identification problem (see, e.g., [4] and the references therein).

In [1] this situation is modeled as an inverse problem for an elliptic equation which is formulated as follows: Let $u(x)$, the voltage, be the solution to the elliptic problem

$$
\begin{aligned}
& \Delta u-p \chi(S) u=0 \quad \text { in } \Omega \subset R^{2}, \\
& \frac{\partial u}{\partial n}=g \geq 0 \text { but } g \not \equiv 0 \text { on } \partial \Omega,
\end{aligned}
$$

where $S \subset \Omega$ and $p>0$ are unknown, $\chi(S)$ is the set characteristic function of $S$, $g$ is the given density of the applied current, $S$ is the location of the contact window, and $p$ is a given positive function of the contact resistivity (e.g., in the following we will assume that $p=R_{s} / \rho_{c}$, with $R_{s}$ the known sheet resistance characterizing the semiconductor layer). We wish to recover $p$ and $S$ from a one-point boundary measurement $u\left(x_{0}\right)$ for some $x_{0} \in \partial \Omega$. In [1] the identifiability of the unknown pair $\{p, S\}$ from a one-parameter family $\{p(t), S(t)\}_{t \in(0,1)}$ is studied, and uniqueness, stability, and continuous dependence results for the one-point measurement on the boundary are obtained.

Received December 20, 1989.

Second author's current address: Center for Applied Mathematical Sciences, University of Southern California, Los Angeles, CA 90089-1113

(C)1991 Brown University 
In this paper, we consider the identification of $p$ when the contact location $S$ is known. Obviously this is a special case of the one-parameter case studied in [1], so we have the uniqueness, stability, and continuous dependence results, but in this simpler case, we have much more detailed properties, as will be shown in Sec. 2. We also establish some quantitative properties for the measurement on the boundary, and apply these to analyze the formula commonly used in industry to extract the contact resistivity from the measurement. We prove that the formula is good only when the true resistivity is large enough, and it overestimates the true resistivity severely when the true resistivity is small. In Sec. 3, based on the properties we develop in Sec. 2, we establish a numerical iteration scheme for identification of the contact resistivity from a given boundary measurement, and prove the convergence of this scheme. We illustrate the use of this scheme with a numerical example in Sec. 4 by using the elliptic PDE solver ELLPACK (see [7]).

We wish to thank Ellis Cumberbatch with whom we had a number of useful discussions concerning the results presented in this paper.

2. The extracted contact resistivity. In applications, in order to identify the contact resistivity $\rho_{c}=R_{s} / p$ in problem (1), (2), the voltage $u$ is measured at some $x_{0} \in$ $\partial \Omega$. Then the following formula is used to extract the contact resistivity, that is, the so-called extracted contact resistivity:

$$
\rho_{c e}=|S| \frac{u\left(x_{0}\right)}{\frac{1}{R_{s}} \int_{\partial \Omega} g d s} .
$$

This formula is based on a simple argument using Ohm's law (see, e.g., $[4,5]$ for details). Computer simulations of this problem are also given in [5]. In this section we study the quantitative behavior of this extracted contact resistivity $\rho_{c e}$ in terms of the true resistivity $\rho_{c}$ as given by (3).

Let $\Omega$ be a bounded connected domain in $R^{2}$ and $S$ a given subdomain in $\Omega$ with $C^{1,1}$ boundaries $\partial \Omega$ and $\partial S$. Points in $R^{2}$ are denoted by $x=\left(x_{1}, x_{2}\right)$. Suppose $g(x) \in C^{\alpha}(\partial \Omega)$ for some $0<\alpha<1$ and $g \geq 0$ but $g \not \equiv 0$.

It is well known that the $H^{1}(\Omega)$ solution to (1), (2) is in $C^{1, \beta}(\Omega)$ for some $0<$ $\beta<1$ (see, e.g., [2]). Denote this solution by $u(x ; p)$. Our identification problem is: recover the positive constant $p$ from the one-point boundary measurement $u\left(x_{0}\right)$, given the geometric setting and the applied current $g$ on the boundary.

Since this is a special case of the one-parameter monotone family studied in [1], we have from there the uniqueness, stability, and continuous dependence of this identification problem. In fact, for this special case we have much better properties and an easier proof.

TheOREM 1. The solution $u(x ; p)$ to $(1),(2)$ is $C^{\infty}$ in $p$, and the $k$ th order derivative with respect to $p$, denoted by $u^{(k)}(x ; p)$, is the solution to

$$
\begin{gathered}
\Delta u^{(k)}-p \chi(S) u^{(k)}=k \chi(S) u^{(k-1)} \text { in } \Omega, \\
\frac{\partial u^{(k)}}{\partial n}=0 \quad \text { on } \partial \Omega,
\end{gathered}
$$


where $k=1,2, \ldots$ and $u^{(0)}=u$. Moreover, we have

$$
(-1)^{k} u^{(k)}(x ; p) \geq 0 \quad \text { in } \bar{\Omega} \quad \text { and } \quad(-1)^{k} u^{(k)}(x ; p)>0 \quad \text { in } \bar{\Omega} \backslash S .
$$

Proof. For a fixed $p>0$, the solution $u=u(x ; p) \in H^{1}(\Omega)$ satisfies

$$
\iint_{\Omega}\{\nabla u \cdot \nabla \phi+p \chi(S) u \phi\} d x=\int_{\partial \Omega} g \phi d s \quad \text { for all } \phi \in H^{1}(\Omega) .
$$

For small $h \neq 0 \quad(|h|<p / 2)$ we set

$$
v_{h}(x ; p)=\frac{u(x ; p+h)-u(x ; p)}{h} .
$$

Then $v_{h}$ is in $H^{1}(\Omega)$ and satisfies

$$
\iint_{\Omega}\left\{\nabla v_{h} \cdot \nabla \phi+(p+h) \chi(S) v_{h} \phi\right\} d x=-\iint_{S} u \phi d x \quad \text { for all } \phi \in H^{1}(\Omega) .
$$

By setting $\phi=v_{h}$ we have

$$
\iint_{\Omega}\left|\nabla v_{h}\right|^{2} d x+(p+h) \iint_{S} v_{h}^{2} d x=-\iint_{S} u v_{h} d x
$$

which implies that

$$
\frac{p}{2} \iint_{S} v_{h}^{2} d x \leq\|u\|_{L^{2}(S)}\left\|v_{h}\right\|_{L^{2}(S)}
$$

so

$$
\left\|v_{h}\right\|_{L^{2}(S)} \leq \frac{2}{p}\|u\|_{L^{2}(S)} .
$$

Notice that the weak form of $(4)_{k}$ is

$$
\iint_{\Omega}\left\{\nabla u^{(k)} \cdot \nabla \phi+p \chi(S) u^{(k)} \phi\right\} d x=-k \iint_{S} u^{(k-1)} \phi d x \quad \text { for all } \phi \in H^{1}(\Omega) \text {, }
$$
$k=1,2, \ldots$. So for $v_{h}-u^{(1)}$ we have

$$
\iint_{\Omega}\left\{\nabla\left(v_{h}-u^{(1)}\right) \cdot \nabla \phi+p \chi(S)\left(v_{h}-u^{(1)}\right) \phi\right\} d x=-h \iint_{S} v_{h} \phi d x
$$

for all $\phi \in H^{1}(\Omega)$. Therefore, setting $\phi=v_{h}-u^{(1)}$ yields

$$
\iint_{\Omega}\left|\nabla\left(v_{h}-u^{(1)}\right)\right|^{2} d x+p \iint_{S}\left(v_{h}-u^{(1)}\right)^{2} d x=-h \iint_{S} v_{h}\left(v_{h}-u^{(1)}\right) d x,
$$

hence

$$
p \iint_{S}\left(v_{h}-u^{(1)}\right)^{2} d x \leq|h| \cdot\left\|v_{h}\right\|_{L^{2}(S)} \cdot\left\|v_{h}-u^{(1)}\right\|_{L^{2}(S)},
$$

i.e., by (7),

$$
\left\|v_{h}-u^{(1)}\right\|_{L^{2}(S)} \leq \frac{|h|}{p}\left\|v_{h}\right\|_{L^{2}(S)} \leq \frac{2|h|}{p^{2}}\|u\|_{L^{2}(S)} .
$$

By the Poincare inequality, we have

$$
C_{1}\|\phi\|_{H^{1}(\Omega)}^{2} \leq\|\nabla \phi\|_{L^{2}(\Omega)}^{2}+\frac{1}{|S|}\left\{\iint_{S} \phi d x\right\}^{2},
$$


hence,

$$
C_{1}\|\phi\|_{H^{1}(\Omega)}^{2} \leq\|\nabla \phi\|_{L^{2}(\Omega)}^{2}+\|\phi\|_{L^{2}(S)}^{2}
$$

for all $\phi \in H^{1}(\Omega)$, where $C_{1}$ is independent of $\phi$. For a proof of the Poincaré inequality as used here, see [9] for the case $S=\Omega$. Notice that the proof given there is also valid for our case $S \subset \Omega$ under the assumptions we have on $S$ and $\Omega$. So (9) yields

$$
C_{1} \cdot \min \{p, 1\} \cdot\left\|v_{h}-u^{(1)}\right\|_{H^{1}(\Omega)}^{2} \leq|h| \cdot\left\|v_{h}\right\|_{L^{2}(S)} \cdot\left\|v_{h}-u^{(1)}\right\|_{L^{2}(S)},
$$

and then by (7), we have

$$
\left\|v_{h}-u^{(1)}\right\|_{H^{1}(\Omega)} \leq C_{p}|h| \cdot\|u\|_{L^{2}(S)},
$$

where $C_{p}$ is a constant independent of $h$.

It is clear that $v_{h}-u^{(1)}$ satisfies

$$
\begin{gathered}
\Delta\left(v_{h}-u^{(1)}\right)=\chi(S)\left((p+h) v_{h}-p u^{(1)}\right) \quad \text { in } \Omega, \\
\frac{\partial\left(v_{h}-u^{(1)}\right)}{\partial n}=0 \text { on } \partial \Omega .
\end{gathered}
$$

Let $G_{0}(x, \xi)$ be a Green's function for the Laplace equation in $\Omega$ with Neumann boundary condition (it is also called the Neumann function, see, e.g., [8]). Then, since $\partial \Omega \cap \bar{S}=\varnothing$, for any $x \in \bar{\Omega}$ we have

$$
v_{h}(x)-u^{(1)}(x)=\iint_{S} G_{0}(x, \xi)\left\{p\left[v_{h}(\xi)-u^{(1)}(\xi)\right]+h v_{h}(\xi)\right\} d \xi+\bar{v}_{h}-\bar{u}^{(1)}
$$

(see, e.g., [8, Chap. 9]), where $\bar{\phi}$ denotes the average of $\phi$ over $\Omega$ :

$$
\bar{\phi}=\frac{1}{|\Omega|} \iint_{\Omega} \phi(x) d x .
$$

Clearly $|\bar{\phi}| \leq\|\phi\|_{L^{2}(\Omega)} / \sqrt{|\Omega|}$. Hence, from (12),

$$
\left|\bar{v}_{h}-\bar{u}^{(1)}\right| \leq C_{p}|h| \cdot\|u\|_{L^{2}(S)} .
$$

Noting that the singularity of $G_{0}$ at $x=\xi$ is square integrable, for $x \in \bar{\Omega}$, from (7), (10), and (14), we have

$$
\begin{aligned}
\left|v_{h}(x)-u^{(1)}(x)\right| & \leq\left\|G_{0}(x, \cdot)\right\|_{L^{2}(S)}\left(p\left\|v_{h}-u^{(1)}\right\|_{L^{2}(S)}+|h| \cdot\left\|v_{h}\right\|_{L^{2}(S)}\right)+\left|\bar{v}_{h}-\bar{u}^{(1)}\right| \\
& \leq|h|\left(\frac{4}{p}\left\|G_{0}(x, \cdot)\right\|_{L^{2}(S)}+C_{p}\right)\|u\|_{L^{2}(S)} \rightarrow 0 \text { as } h \rightarrow 0 .
\end{aligned}
$$

Therefore, $u^{(1)}(x ; p)$ is the derivative of $u(x ; p)$ with respect to $p$ for each $x \in \bar{\Omega}$. The same argument applies to show that $u^{(k)}(x ; p)$ satisfying $(4)_{k}$ is the $k$ th order derivative of $u(x ; p)$ with respect to $p, k=2,3, \ldots$.

To prove $(5)_{k}$, we consider the equation

$$
\Delta w-p \chi(S) w=\chi(S) F \quad \text { in } \Omega
$$


with homogeneous Neumann condition on $\partial \Omega$, where $F$ is continuous in $\Omega$. It is known that $w \in C^{1}(\bar{\Omega})$. By applying the maximum principle for elliptic equations (see, e.g., [6]), we can obtain the following conclusion:

$$
F \leq 0 \text { in } S \text { implies } w \geq 0 \text { in } \bar{\Omega} \text { and } w>0 \text { in } \bar{\Omega} \backslash S .
$$

From Lemma 2.1 in [1], we have $u \geq 0$ in $\bar{\Omega}$. Therefore, we can establish $(5)_{k}$ by applying a simple induction on $n$ and (15). Thus the proof is completed.

RemarK. The Green's function $G_{0}$ we chose in the proof is unique up to an arbitrary constant, but expression (13) does not depend on the choice of the constant. We can see this by setting $\phi \equiv 1$ in (8).

Next, we study the quantitative behavior of the extracted contact resistivity given by (3). For simplicity, we set $R_{S}=1$, i.e., $\rho_{c}=1 / p$. Then the extracted contact resistivity $\rho_{c e}$ is a function of the true $\rho_{c}$ given by

$$
\rho_{c e}=f\left(\rho_{c}\right) \equiv \frac{|S|}{\int_{\partial \Omega} g d s} u\left(x_{0} ; \frac{1}{\rho_{c}}\right),
$$

where $u(x ; p)$ is the solution to (1), (2) and $x_{0}$ is a point at $\partial \Omega$ where the measurement is made. For the so-called Kelvin resistor (a special choice of the density distribution $g$ and the measurement location $x_{0}$ ), some asymptotic properties of $\rho_{c e}$ are observed in [5] by using computer simulations. In general, we have the following properties for $f(\cdot)$ which agree with the observations made in [5]. Let

$$
A_{0}=\frac{\int_{\partial \Omega} g d s}{|S|}>0 \text {. }
$$

THEOREM 2. The function $f(\cdot)$ given by (16) has the following properties:

(i) $f(\cdot)$ is in $C^{\infty}(0, \infty)$ and is strictly increasing in $(0, \infty)$.

(ii) As $\rho_{c} \rightarrow \infty$,

(iii)

$$
\frac{f\left(\rho_{c}\right)}{\rho_{c}}=1+O\left(\frac{1}{\rho_{c}}\right) \text {. }
$$

$$
\lim _{\rho_{c} \rightarrow 0+} f\left(\rho_{c}\right)=f_{0}=\frac{|S|}{\int_{\partial \Omega} g d s} u_{0}\left(x_{0}\right)>0,
$$

where $u_{0}(x)$ is the solution to

$$
\left\{\begin{aligned}
\Delta u_{0}=0 & \text { in } \Omega \backslash \bar{S} \\
u_{0}=0 & \text { on } \partial S \\
\partial u_{0} / \partial n=g & \text { on } \partial \Omega .
\end{aligned}\right.
$$

Proof. (i) follows from Theorem 1 directly.

(ii) Let $v(x ; p)=u(x ; p)-A_{0} / p$. Then we have

$$
v\left(x_{0} ; \frac{1}{\rho_{c}}\right)=A_{0}\left(f\left(\rho_{c}\right)-\rho_{c}\right)
$$

and $v(x ; p)$ satisfies

$$
\begin{aligned}
\Delta v-p \chi(S) v & =A_{0} \chi(S) \quad \text { in } \Omega, \\
\partial v / \partial n & =g \quad \text { on } \partial \Omega .
\end{aligned}
$$


Notice that setting $\phi=1$ in (6) leads to

$$
p \iint_{S} u(x ; p) d x=\int_{\partial \Omega} g d s .
$$

Therefore

$$
\iint_{S} v(x ; p) d x=0
$$

and the Poincare inequality (11) applied to $v$ gives

$$
C\|v\|_{L^{2}(\Omega)} \leq\|\nabla v\|_{L^{2}(\Omega)},
$$

or, equivalently,

$$
C\|v\|_{H^{1}(\Omega)} \leq\|\nabla v\|_{L^{2}(\Omega)},
$$

where $C>0$ depends only on $S$ and $\Omega$. In the weak form of (19), that is,

$$
\iint_{\Omega}\{\nabla v \cdot \nabla \phi+p \chi(S) v \phi\} d x=\int_{\partial \Omega} g \phi d s-A_{0} \iint_{S} \phi d x \quad \text { for all } \phi \in H^{1}(\Omega),
$$

we set $\phi=v$ and use (20) to obtain

$$
\|\nabla v\|_{L^{2}(\Omega)}^{2}=\iint_{\Omega}|\nabla v|^{2} d x \leq \int_{\partial \Omega} g v d s \leq C\|v\|_{H^{1}(\Omega)},
$$

where the trace embedding $H^{1}(\Omega) \hookrightarrow L^{2}(\partial \Omega)$ is used in the second inequality, and $C>0$ is a constant independent of $p$. Hence, by (21) we have

$$
\|v\|_{L^{2}(\Omega)} \leq\|v\|_{H^{\prime}(\Omega)} \leq C .
$$

From (19), for $x \in \Omega$,

$v(x ; p)=-\int_{\partial \Omega} G_{0}(x, \xi) g(\xi) d \xi+A_{0} \iint_{S} G_{0}(x, \xi) d \xi+p \iint_{S} G_{0}(x ; \xi) v(\xi ; p) d \xi+\bar{v}$,

where $G_{0}$ is the Green's function defined in the proof of Theorem 1 (see, e.g., [8, Chap. 9]). By the continuity of $v$ up to $\partial \Omega$ and the integrable singularity of $G_{0}(x, \xi)$ at $x=\xi,(23)$ is also true for $x \in \partial \Omega$. Therefore, from (22) it easily follows that

$$
\left|v\left(x_{0} ; p\right)\right| \leq C_{1}+C_{2} p \quad \text { for any } p>0,
$$

with $C_{1}, C_{2}$ independent of $p$. That is, $v\left(x_{0} ; p\right)=O(1)$ as $p \rightarrow 0$. So we have (ii) by (18).

(iii) Setting $\phi=u$ in (6) yields

$$
\iint_{\Omega}\left\{|\nabla u|^{2}+p \chi(S) u^{2}\right\} d x=\int_{\partial \Omega} g u d s \leq C_{2}\|u\|_{H^{\prime}(\Omega)},
$$

where the trace theorem is used. Hence, by (11),

$$
C_{1}\|u\|_{H^{1}(\Omega)}^{2}+(p-1)\|u\|_{L^{2}(S)}^{2} \leq C_{2}\|u\|_{H^{1}(\Omega)}
$$

for $p>2$. That is,

$$
C_{1}\left(\|u\|_{H^{1}(\Omega)}-\frac{C_{2}}{2 C_{1}}\right)^{2}+(p-1)\|u\|_{L^{2}(S)}^{2} \leq \frac{C_{2}^{2}}{4 C_{1}} .
$$


Hence

Also from (24), we have

$$
\|u\|_{L^{2}(S)}^{2} \leq \frac{C_{2}^{2}}{4 C_{1}(p-1)} \leq \frac{C}{p} .
$$

$$
\|u\|_{H^{1}(S)} \leq \frac{C_{2}}{C_{1}} .
$$

For a fixed $\varepsilon \in(0,1 / 2), H^{1-\varepsilon}(S)$ is defined as the interpolation space between $H^{0}(S)=L^{2}(S)$ and $H^{1}(S)$ (see e.g., [3, Chap. 1, Sec. 9]) and there holds the interpolation inequality

$$
\|w\|_{H^{1-\varepsilon}(S)} \leq C\|w\|_{H^{0}(S)}^{\varepsilon} \cdot\|w\|_{H^{1}(S)}^{1-\varepsilon}
$$

for any $w \in H^{1}(S)$, where $C$ depends only on $S$ and $\varepsilon$. Combining (25), (26), and (27), we have

$$
\|u\|_{H^{1-\varepsilon}(S)} \leq \frac{C}{p^{\varepsilon / 2}} .
$$

Then the trace embedding in $H^{1-\varepsilon}(S)$ gives

$$
H^{1-\varepsilon}(S) \hookrightarrow H^{1 / 2-\varepsilon}(\partial S) \hookrightarrow H^{0}(\partial S)=L^{2}(\partial S)
$$

since $0<\varepsilon<\frac{1}{2}$ (see, e.g., [3]). Hence

$$
\|u\|_{L^{2}(\partial S)} \leq \frac{C}{p^{\varepsilon / 2}} .
$$

Notice that $u-u_{0}\left(u_{0}\right.$ given by (17)) satisfies

$$
\left\{\begin{aligned}
\Delta\left(u-u_{0}\right)=0 & \text { in } \Omega \backslash \bar{S} \\
u-u_{0}=u & \text { on } \partial S \\
\partial\left(u-u_{0}\right) / \partial n=0 & \text { on } \partial \Omega
\end{aligned}\right.
$$

Let $G(x, \xi)$ be the Green's function of the Laplace equation in $\Omega \backslash \bar{S}$ with Neumann boundary condition on $\partial \Omega$ and Dirichlet on $\partial S$. Then for $x \in \Omega$,

$$
u(x ; p)-u_{0}(x)=\int_{\partial S} \frac{\partial G}{\partial n}(x, \xi) u(\xi ; p) d \xi .
$$

Since $\operatorname{dist}(\partial \Omega, \partial S)>0, \partial G / \partial n(x, \cdot)$ is regular on $\partial S$ for each $x \in \partial \Omega$. Also $G$ is independent of $p$. Therefore, (29) is true for $x \in \partial \Omega$, and by (28)

$$
\left|u(x ; p)-u_{0}(x)\right| \leq C\|u\|_{L^{2}(\partial S)} \leq \frac{C}{p^{\varepsilon / 2}},
$$

i.e., for each $x \in \partial \Omega$,

$$
u(x ; p) \rightarrow u_{0}(x) \text { as } p \rightarrow \infty .
$$

Applying the maximum principle to $u_{0}$ easily yields $u_{0}>0$ on $\partial \Omega$. Thus (iii) is proved.

From (ii) of this theorem, we can see that this extracted resistivity is a good estimate when the true resistivity $\rho_{c}$ is large. However, from (iii) it is seen that for small $\rho_{c}$ the extracted resistivity overestimates $\rho_{c}$ severely, since the limiting value $f_{0}$ of $\rho_{c e}$ as $\rho_{c} \rightarrow 0$ in (iii) is a positive constant. Also (iii) provides a method to calculate $f_{0}$. For physical interpretations of these properties, see [5]. 
3. Identification of the contact resistivity. In this section, we construct a numerical iteration scheme for identification of the contact resistivity $\rho_{c}$, or, equivalently, the constant $p$, from the one-point boundary measurement. Assume the geometric settings of the problem (1), (2) are given, i.e., $\Omega$, the contact window $S$, the point $x_{0} \in \partial \Omega$ where the measurement is made, and the applied current density $g$ are given. Then we wish to estimate the constant $p^{*}$ from the measurement $u^{*}$.

From Theorem $1, u\left(x_{0} ; p\right)$ is strict decreasing in $p$ and is convex. So for each measurement $u^{*}>u_{0}\left(x_{0}\right) \quad\left(u_{0}(x)\right.$ is given by (17)) there is a unique $p^{*}>0$ such that $u^{*}=u\left(x_{0} ; p^{*}\right)$, where $u(x ; p)$ is the solution to (1), (2). To ensure unique identifiability, in the following we assume that $u^{*}>u_{0}\left(x_{0}\right)$.

By Newton's method of finding a zero of a function, we construct the following iteration scheme:

$$
p_{j+1}=p_{j}-\frac{u\left(x_{0} ; p_{j}\right)-u^{*}}{u^{(1)}\left(x_{0} ; p_{j}\right)},
$$

where $u\left(x ; p_{j}\right)$ is the solution to $(1),(2)$ with $p=p_{j}$, and $u^{(1)}\left(x ; p_{j}\right)$ is the derivative of $u(x ; p)$ with respect to $p$ given by $(4)_{1}$ with $p=p_{j}$.

Besides the quadratic convergence property of the general Newton's method, for this specific problem, we have

Theorem 3. If the initial guess $p_{0}>0$ is such that $u\left(x_{0} ; p_{0}\right)>u^{*}$, then the sequence $\left\{p_{j}\right\}_{0}^{\infty}$ given by (30) is strictly increasing and convergent from below to $p^{*}$, the unique value such that $u^{*}=u\left(x_{0} ; p^{*}\right)$.

Proof. First we prove the strict monotonicity. For $j=0$,

$$
p_{1}=p_{0}-\frac{u\left(x_{0} ; p_{0}\right)-u^{*}}{u^{(1)}\left(x_{0} ; p_{0}\right)}>p_{0}
$$

since $u^{(1)}$ is negative and by the assumption $u\left(x_{0} ; p_{0}\right)>u^{*}$. For $j \geq 1$, from $(30)_{j-1}$,

$$
u^{*}=u\left(x_{0} ; p_{j-1}\right)+\left(p_{j}-p_{j-1}\right) u^{(1)}\left(x_{0} ; p_{j-1}\right),
$$

therefore, for $(30)_{j}$,

$$
\begin{aligned}
\left(p_{j+1}-p_{j}\right) u^{(1)}\left(x_{0} ; p_{j}\right) & =u^{*}-u\left(x_{0} ; p_{j}\right) \\
& =-\left\{u\left(x_{0} ; p_{j}\right)-u\left(x_{0} ; p_{j-1}\right)\right\}+\left(p_{j}-p_{j-1}\right) u^{(1)}\left(x_{0} ; p_{j-1}\right) \\
& =-\frac{\left(p_{j}-p_{j-1}\right)^{2}}{2} u^{(2)}\left(\xi_{j} ; p_{j-1}\right)
\end{aligned}
$$

$\left(\xi_{j}\right.$ is between $p_{j}$ and $\left.p_{j-1}\right)$. By Theorem 1 , we have $u^{(1)}\left(x_{0} ; p\right)<0$ and $u^{(2)}\left(x_{0} ; p\right)$ $>0$, hence, from above, $p_{j+1}>p_{j}$ for $j=1,2, \ldots$. Thus the sequence $\left\{p_{j}\right\}_{0}^{\infty}$ is strictly increasing.

Therefore $p_{j} \uparrow \tilde{p}$ for some $\tilde{p}>0$ as $j \rightarrow \infty$. Letting $j$ tend to $\infty$ in (30), we have $u\left(x_{0} ; \tilde{p}\right)=u^{*}$. Hence $\tilde{p}=p^{*}$ by the strict monotonicity of $u$ in $p$. Thus the roof is completed. 
Next we consider an appropriate choice of the initial guess $p_{0}$. We call $p_{0}$ an eligible initial value if $u\left(x_{0} ; p_{0}\right) \geq u^{*}$, or, equivalently, $p_{0} \leq p^{*}$. So if $p_{0}$ is eligible, by Theorem 3 the Newton iteration scheme (30) starting at this $p_{0}$ is monotonely increasing and converges to $p^{*}$. On the other hand, if $p_{0}$ is not eligible, i.e., $u\left(x_{0} ; p_{0}\right)<u^{*}$, then $p_{0}>p^{*}$, and clearly the next iteration $p_{1}$ by (30) may become nonpositive, so the iteration cannot proceed. In this case, instead of using (30) to obtain the next iteration, we set $p_{1}=p_{0} / 4$. We can continue this procedure until we come up with a $p_{i}$ such that $u\left(x_{0} ; p_{i}\right)>u^{*}$, then we turn to the Newton scheme (30) for the rest of the iterations, using this $p_{i}$ as the initial value. At each iteration step, two elliptic problems, one for $u$ and one for $u^{(1)}$, need to be solved to obtain the next iteration value.

We remark that we can also use the bisection method to identify $p^{*}$. For this method we need to search for the interval on which we start the bisection procedure. Similar ideas as in the search for the eligible initial value $p_{0}$ above can be used to find this interval. At each iteration step, only one elliptic problem (for $u$ itself) needs to be solved.

Comparing these two schemes, we notice that we compensate the speed of convergence in the bisection method for the ease of solving only one elliptic problem.

Finally, we remark that in the above we assume that there is no noise in the measurement. In the case that there is noise in the measurement but the experiment is repeated for a number of times, we should take the mean value for these data first and then use the above scheme to find the $p$ corresponding to this mean value as the estimate for the true $p$.

4. A numerical example. In this section we consider an example where $\Omega$ and $S$ are two nonconcentric discs. Let

$$
\Omega=\text { the unit disc }, \quad S=\text { a disc centered at }(0,0.2) \text { with radius } \frac{1}{2} .
$$

The applied current density is given by

$$
g(x)= \begin{cases}\left|x_{2}+\frac{1}{2}\right| & \text { when } x_{2} \leq-\frac{1}{2}, \\ 0 & \text { when } x_{2}>-\frac{1}{2},\end{cases}
$$

and the measurement is made at $x_{0}=(1,0)$.

For any given $u^{*}$, we pick an arbitrary initial $p_{0}$. Then we solve (1), (2) with this $p_{0}$ and test if the $p_{0}$ is eligible. If not, we reduce to a quarter of $p_{0}$ and test again, until we get an eligible initial value. Once we get an eligible $p_{0}$, we use the Newton iteration scheme (30) to obtain the next value, until the present value is close enough to the previous one, or the calculated $u$ value is close enough to the measured $u^{*}$. At each iteration step, we use the elliptic problem solver called ELLPACK (see [7]) to solve for $u\left(x ; p_{j}\right)$ and $u^{(1)}\left(x ; p_{j}\right)$. Notice that in the problem for $u^{(1)}\left(x ; p_{j}\right)$, that is, $(4)_{1}$, we need the values of $u\left(x ; p_{j}\right)$. The result is given in Table 1 . For comparison we use the bisection method shown in Table 2. Notice that the same problem takes Newton's method eight iteration steps (i.e., solve 16 elliptic problems) to converge while the bisection method takes 21 steps (solve 21 elliptic problems). 
TABLE 1. Newton's method with $p_{0}=2.0$ and $u^{*}=1.0$.

The estimated $p^{*}=0.971166$.

\begin{tabular}{|c|c|c|}
\hline$j$ & $p_{j}$ & $u\left(x_{0} ; p_{j}\right)$ \\
\hline 0 & 2.000000 & 0.527925 \\
\hline 1 & 0.500000 & 1.863598 \\
\hline 2 & 0.734298 & 1.295691 \\
\hline 3 & 0.904528 & 1.067542 \\
\hline 4 & 0.962841 & 1.007927 \\
\hline 5 & 0.970565 & 1.000568 \\
\hline 6 & 0.971127 & 1.000037 \\
\hline 7 & 0.971164 & 1.000002 \\
\hline 8 & 0.971166 & 1.000000 \\
\hline
\end{tabular}

TABLE 2. Bisection method with $p_{0}=2.0$ and $u^{*}=1.0$.

The estimated $p^{*}=0.971166$.

\begin{tabular}{|c|c|c|}
\hline$j$ & $p_{j}$ & $u\left(x_{0} ; p_{j}\right)$ \\
\hline 0 & 2.000000 & 0.527925 \\
\hline 1 & 1.000000 & 0.973563 \\
\hline 2 & 0.500000 & 1.863598 \\
\hline 3 & 0.750000 & 1.270314 \\
\hline 4 & 0.875000 & 1.100757 \\
\hline 5 & 0.937500 & 1.032924 \\
\hline 6 & 0.968750 & 1.002287 \\
\hline 7 & 0.984375 & 0.987697 \\
\hline 8 & 0.976563 & 0.994934 \\
\hline 9 & 0.972656 & 0.998596 \\
\hline 10 & 0.970703 & 1.000437 \\
\hline
\end{tabular}

\begin{tabular}{|c|c|c|}
\hline$j$ & $p_{j}$ & $u\left(x_{0} ; p_{j}\right)$ \\
\hline 11 & 0.971680 & 0.999516 \\
\hline 12 & 0.971191 & 0.999976 \\
\hline 13 & 0.970947 & 1.000207 \\
\hline 14 & 0.971069 & 1.000092 \\
\hline 15 & 0.971130 & 1.000034 \\
\hline 16 & 0.971161 & 1.000005 \\
\hline 17 & 0.971176 & 0.999991 \\
\hline 18 & 0.971169 & 0.999998 \\
\hline 19 & 0.971165 & 1.000002 \\
\hline 20 & 0.971167 & 0.999999 \\
\hline 21 & 0.971166 & 1.000000 \\
\hline
\end{tabular}




\section{REFERENCES}

[1] W. Fang and E. Cumberbatch, Inverse problems for MOSFET contact resistivity, SIAM J. Appl. Math., to appear

[2] O. A. Ladyzhenskaya and N. N. Ural'tzeva, Linear and Quasilinear Elliptic Equations, Academic Press, London, 1968

[3] J. L. Lions and E. Magenes, Non-Homogeneous Boundary Value Problems and Applications. I, Springer-Verlag, Berlin-Heidelberg, 1970

[4] W. H. Loh, Modelling and measurement of contact resistance, Stanford Electronic Labs., Tech. Rep., No. G830-1, Dec. 1987

[5] W. H. Loh, K. Saraswat, and R. W. Dutton, Analysis and scaling of Kelvin resistors for extraction of specific contact resistivity, IEEE Electrion Device Letters 6 (3), 105-108 (1985)

[6] M. H. Protter and H. F. Weinberger, Maximum Principles in Differential Equations, SpringerVerlag, New York, 1984

[7] J. Rice and R. F. Boisvert, Solving Elliptic Problems Using ELLPACK, Springer-Verlag, New York, 1985

[8] G. F. Roach, Green's Functions, 2nd ed., Cambridge Univ. Press, Cambridge, 1982

[9] J. Wloka, Partial Differential Equations, Cambridge Univ. Press, Cambridge, 1987 